\title{
Magnetic Resonance Imaging and GeneXpert: A Rapid and Accurate Diagnostic Tool for the Management of Tuberculosis of the Spine
}

\author{
Ayush Sharma $^{1,2}$, Harvinder Singh Chhabra ${ }^{2}$, Rajat Mahajan ${ }^{2}$, Tarun Chabra ${ }^{2}$, Sahil Batra ${ }^{2}$ \\ ${ }^{1}$ Department of Orthopedic and Spine Surgery, Dr. Babasaheb Ambedkar Central Railway Hospital, Mumbai, India \\ ${ }^{2}$ Department of Spine Services Indian Spinal Injuries Center, New Delhi, India
}

Study Design: Retrospective study.

Purpose: The aim of this study was to analyze various diagnostic tools, including GeneXpert, for the management of tuberculosis of the spine.

Overview of Literature: Traditional diagnostic methods of microscopy, histology, and culture have low sensitivity and specificity for the management of tuberculosis of the spine.

Methods: Of the 262 treated cases of spinal tuberculosis, data on 1 year follow-up was available for 217 cases. Of these, only 145 cases with a confirmed diagnosis were selected for retrospective analysis.

Results: In 145 of the 217 patients (66.80\%), diagnosis was confirmed on the basis of a culture. Of the 145 patients with a confirmed diagnosis, 98 (66.20\%) patients were diagnosed on the basis of clinical presentation, whereas $123(84.8 \%)$ exhibited a typical magnetic resonance imaging (MRI) picture. In 99 surgically treated patients, the diagnosis was confirmed on the basis of an intraoperative tissue biopsy. Among the 46 patients treated conservatively, 35 underwent a transpedicular biopsy, 4 patients underwent computed tomography-guided biopsy, 6 patients were diagnosed on the basis of material obtained from a cold abscess, and 1 patient underwent an open biopsy. The sensitivity of the culture for the detection of Mycobacterium tuberculosis was 66.80\% (145/217) in our patients. Among the cases in which GeneXpert was used, the sensitivity for the detection of Mycobacterium tuberculosis was 93.4\% (43/46). Moreover, the sensitivity of GeneXpert to detect rifampicin resistance was 100\% (7/7) in our study.

Conclusions: Majority of the patients with tuberculosis of the spine can be diagnosed on the basis of a typical radiological presentation via MRI. In our study, $84.8 \%$ cases exhibited typical MRI findings. For patients presenting with atypical MRI features, a rapid and accurate diagnosis is possible by combining GeneXpert with MRI. The combined use of MRI and GeneXpert is a rapid and highly sensitive tool to diagnose tuberculosis and rifampicin resistance in patients with tuberculosis of the spine. Furthermore, we achieved a $97.9 \%$ sensitivity for the detection of Mycobacterium tuberculosis and 100\% sensitivity for the detection of rifampicin resistance in our study.

Keywords: GeneXpert; Tuberculosis; Diagnosis; Spine

Received Jan 10, 2016; Revised Feb 9, 2016; Accepted Feb 24, 2016

Corresponding author: Ayush Sharma

Doctor's quarter no 6, Dr B R Ambedker Central Railway Hospital, Byculla east, Mumbai, India 400027

Tel: +91-90-0454-9623, E-mail: drayush@gmail.com 


\section{Introduction}

Musculoskeletal affection is observed in $4 \%$ of all cases with tuberculosis; $50 \%$ of which involve the spine [1], which is the most common form of skeletal tuberculosis. Currently, the diagnosis of tuberculosis of the spine is primarily based on clinico-radiological observations. A typical presentation of tuberculosis of the spine consists of pain during movement with a localized deformity in the back that is tender following percussion as well as other typical systemic symptoms of active tuberculosis (i.e., night cries, malaise, weight loss, loss of appetite, night sweats, and a rise in temperature in the evening). Moreover, patients may or may not have a neurological deficit, which can be the first symptom in rare cases [1]. Microscopic confirmation using Ziehl-Nielsen staining remains a popular diagnostic method because of its simplicity and cost-effectiveness; however, it has a low sensitivity and requires $10,000-100,000$ bacilli $/ \mathrm{mL}$ in clinical specimens to be positive [2,3]. Mycobacterium tuberculosis (Mtb) culture is the gold standard method for the diagnosis of tuberculosis, but it also has various limitations, including a required 6-8 week period of growth because of the slow replication rate of the bacteria; these results are often negative as it requires $10-100 \mathrm{bacilli} / \mathrm{mL}$ (live bacilli) in clinical specimens to achieve culture positive results $[4,5]$. Magnetic resonance imaging (MRI) is a better diagnostic method than radiography [6]. Marrow edema, endplate disruption, paravertebral soft tissue formation, subligamentous collections, and a high signal of the intervertebral disc on T2-weighted are typical MRI features with good to excellent sensitivity for spinal tuberculosis. Overall, the sensitivity and specificity of MRI for spinal tuberculosis are $100 \%$ and $88.2 \%$, respectively $[7,8]$. MRI findings with a high sensitivity and specificity include the disruption of the end-plate (100\% and $81.4 \%$, respectively), paravertebral soft-tissue shadow ( $96.8 \%$ and $85.3 \%$, respectively), and high signal intensity of the intervertebral disc on the T2-weighted image ( $80.6 \%$ and $82.4 \%$, respectively). Atypical presentation primarily includes discrete foci of spinal involvement with intervening normal vertebrae and no evidence of a connecting soft tissue abscess or any other MRI features typical of tuberculosis as discussed above (i.e., the involvement of only the posterior column of the spine without end plate involvement and multiple skip lesions without a soft tissue shadow). In patients with an atypical clinical and MRI presentation, further inves- tigation in the form of an open or precautionary biopsy can be performed to confirm the diagnosis. Although the specimen adequacy is higher for an open biopsy than for a percutaneous biopsy, the similarity ratio between the initial radiological and final pathological diagnosis of both techniques are favorable (71.4\% for the open biopsy and 69.2\% for the percutaneous biopsy) [9]. Recent techniques, such as polymerase chain reaction (PCR) and GeneXpert provide improved accuracy over microscopy and are more rapid than bacterial cultures. The GeneXpert test has a sensitivity of $95.6 \%$ and a specificity of $96.2 \%$ for diagnosis of spinal tuberculosis [10].

Additionally, GeneXpert is more likely to detect Mtb DNA than traditional PCR, with the added advantage of also determining rifampicin resistance [11]. A delay in diagnosis and the failure to detect drug resistance are major hurdles involved in the treatment of tuberculosis of the spine even today. The aim of this study was to analyze various diagnostic tools for the management of tuberculosis of the spine, particularly the use of rapid diagnostic tools, such as GeneXpert and MRI.

\section{Materials and Methods}

All cases with tuberculosis of the spine treated at the Indian Spinal Injuries Center (New Delhi), a tertiary care spine and rehabilitation center, between October 2012 and December 2014 were retrospectively analyzed. October 2012 was used as the cutoff as this was the time our center initiated the use of GeneXpert as a diagnostic tool for tuberculosis of the spine. Of the 262 cases during this period, data on a follow-up of more than 1 year was available for 217 cases. Of these 217 cases, only 145 (66.80\%) cases were bacteriologically confirmed via a bacterial culture and were included in the present study. One of the authors independently analyzed the MRI films, reports, and history of all the patients and categorized them into typical and atypical cases (Figs. 1A, 2A). All cases that did not exhibit a typical presentation as discussed above were categorized as "atypical." Diagnostic biopsy methods and the use of GeneXpert were also studied. Data were analyzed according to the documented American Spinal Injury Association (AIS) impairment scale [12] at the time of presentation and at the final follow-up to determine the extent of neurological recovery. All cases that presented with a neurological deficit were divided into three groups: (1) completely improved (AIS grade at the final follow-up 

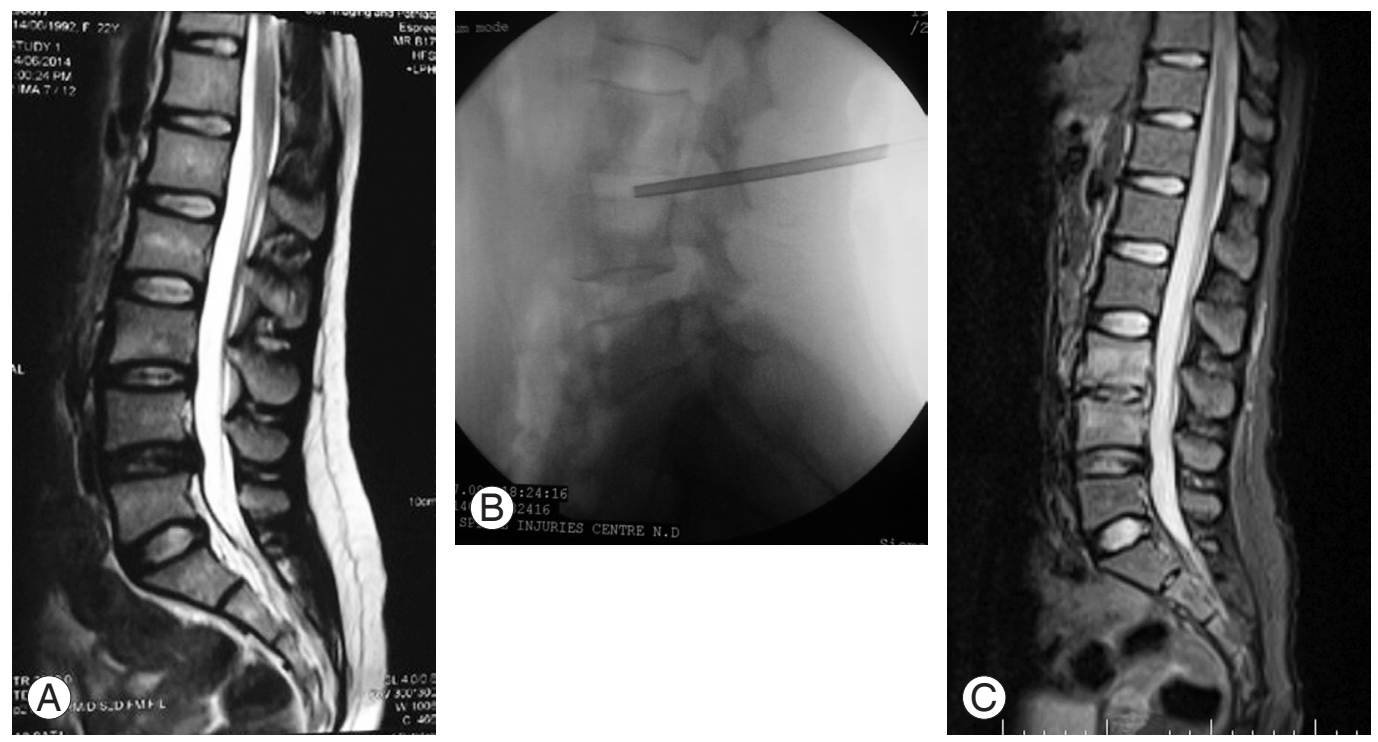

Fig. 1. An atypical case of tuberculosis of the spine. A 22-year-old female patient presented with only back pain. (A) Atypical magnetic resonance imaging (MRI) presentation via the T2-weighted image. (B) Percutaneous intradiscal biopsy was performed from the lumber 3-4 disc, and GeneXpert was used for diagnostic confirmation. (C) Follow-up MRI revealed the T2-weighted image after conservative treatment showing resolution of the disease.
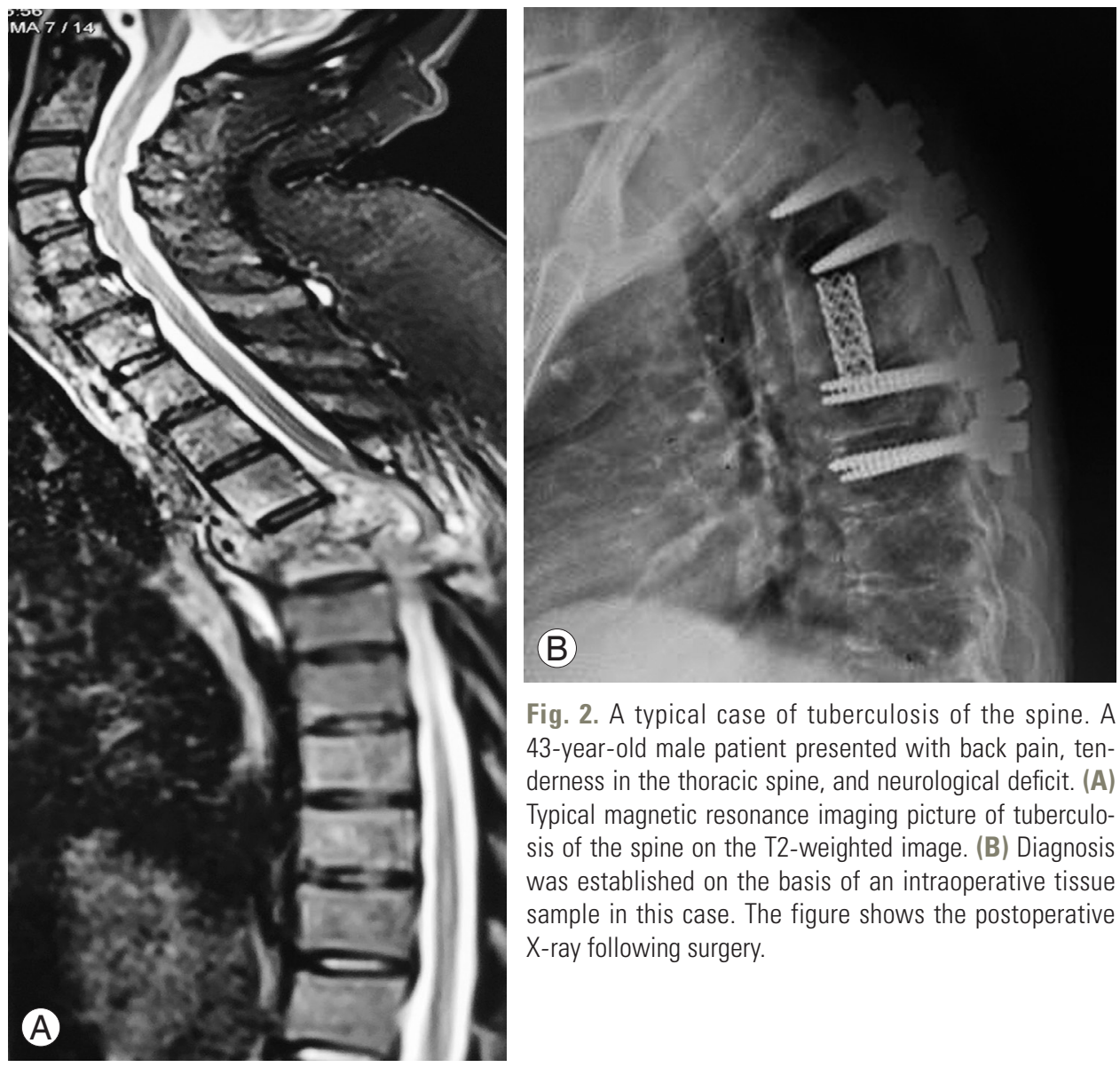

Fig. 2. A typical case of tuberculosis of the spine. A 43-year-old male patient presented with back pain, tenderness in the thoracic spine, and neurological deficit. (A) Typical magnetic resonance imaging picture of tuberculosis of the spine on the T2-weighted image. (B) Diagnosis was established on the basis of an intraoperative tissue sample in this case. The figure shows the postoperative $\mathrm{X}$-ray following surgery. 
was E), (2) partially improved (AIS grade changed to B, C, or $\mathrm{D}$ but not $\mathrm{E}$ ), and (3) no improvement (AIS grading at presentation and final follow-up remained unchanged).

\section{Results}

The average patient age was $45.8 \pm 19.1$ years, and 71 male and 74 female patients were included. A total of 98 (66.20\%) patients exhibited the typical clinical presentation, whereas $123(84.8 \%)$ presented with the typical MRI findings as discussed above. There were 99 patients who were surgically treated, whereas 46 patients were managed conservatively (Figs. 1C, 2B). In all of the surgically treated cases, the diagnosis was made on the basis of an intraoperative tissue biopsy obtained from the diseased vertebra and intervening disc space. In the 46 patients managed conservatively, 35 patients underwent a transpedicular or intradiscal percutaneous biopsy (Fig. 1B), 4 patients underwent a computed tomography (CT)-guided biopsy; 6 patients were diagnosed on the basis of material obtained from cold abscess and 1 patient underwent open biopsy (Fig. 3).

GeneXpert was used in 46 cases, from which 23 tissue samples were obtained from an intraoperative biopsy, 18 were attained via a transpedicular biopsy, 3 were obtained from the aspiration of a cold abscess, and 1 was obtained from CT-guided and open biopsies. Of the 43 cases that tested positive using GeneXpert, seven also tested positive for rifampicin resistance (Fig. 4). The patients that tested positive for rifampicin resistance were further confirmed by a drug sensitivity test. Although the sensitivity of GeneXpert for the detection of rifampicin resistance was 100\% (7/7) in our study, the overall sensitivity for the detection of Mtb was 93.4\% (43/46). In addition, GeneXpert was used in all 22 patients with an atypical MRI to confirm the diagnosis. In the 44 patients who were neurologically intact at presentation, no neurological deterioration was observed at the final follow-up after the administration of appropriate conservative or surgical treatment. Following treatment, among the 101 patients who presented with a neurological deficit according to the AIS grading system, 70 (69.30\%) exhibited a complete neurological improvement, 21 (20.7\%) underwent a partial improvement, and $10(9.9 \%)$ patients showed no improvement at the final follow-up (Fig. 5).

\section{Discussion}

A delay in both the diagnosis and initiation of treatment as well as the failure to recognize cases of drug resistance could have an adverse effect on the prognosis of patients with tuberculosis of the spine $[13,14]$. Traditional diagnostic methods are based on the typical clinical features followed by a bacteriological confirmation via positive histology and culture. Moreover, the traditional methods of microscopy, histology, and culture have a low sensitivity

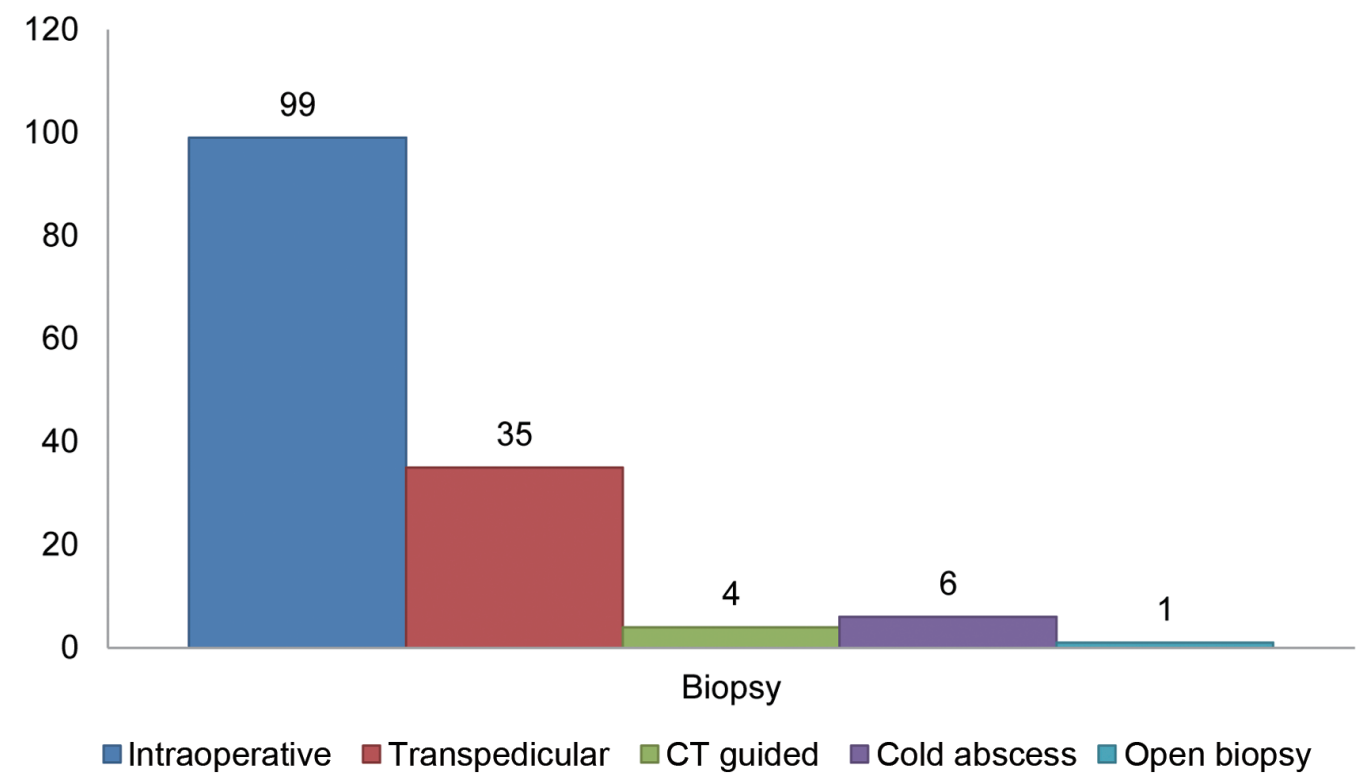

Fig. 3. A graph showing the type of biopsy (X-axis) and number of patients (Y-axis). CT, computed tomography. 


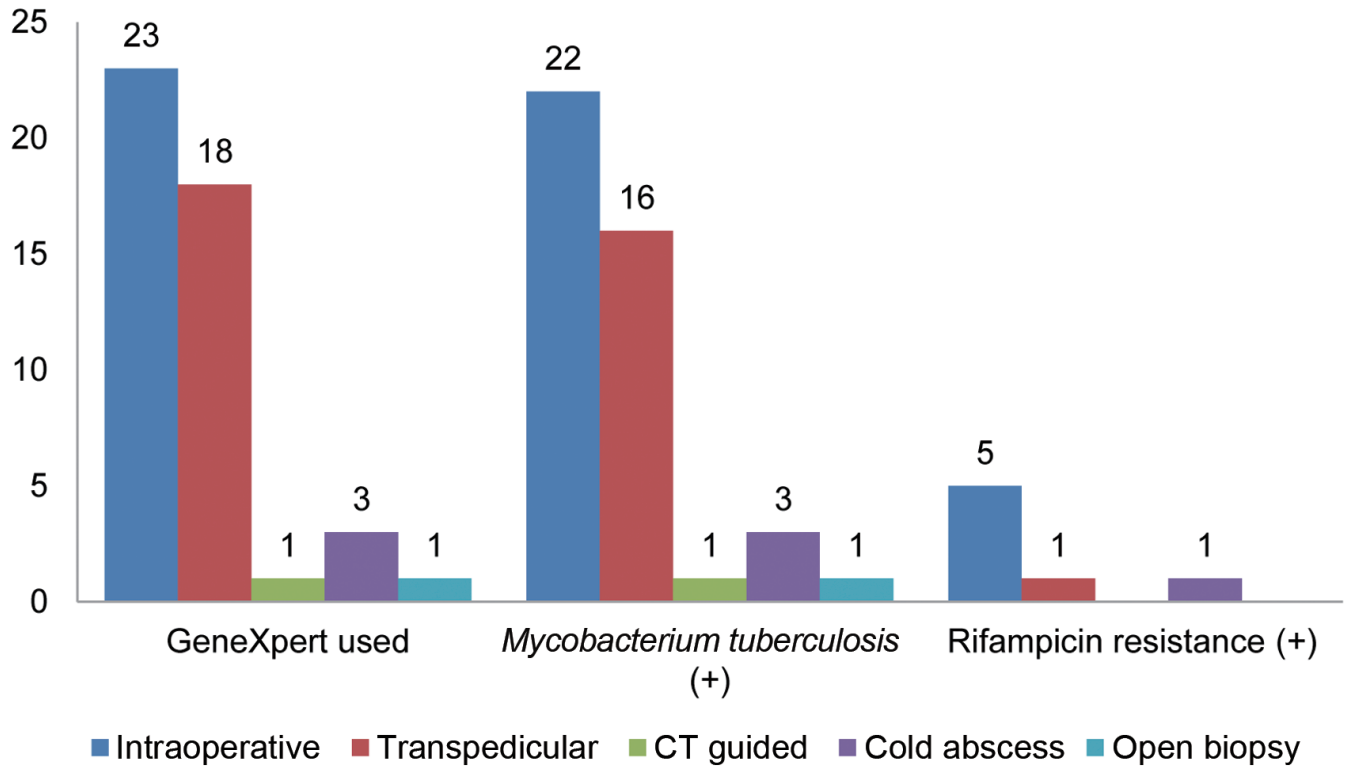

Fig. 4. A graph showing whether GeneXpert was used, if Mycobacterium tuberculosis was detected, the presence of rifampicin resistance (X-axis), and the number of patients (Y-axis).



Fig. 5. A graph of the neurological improvement (X-axis) and the number of patients (Y-axis).

and specificity. The sensitivity of histology to confirm a diagnosis of spinal tuberculosis has been reported to be approximately $60 \%$. In addition, the incidence of positive cultures for acid-fast bacilli in osteoarticular tuberculous lesions has been reported to be between $40 \%$ and $88 \%$ [15-18]. In our present study, to confirm the diagnosis of tuberculosis of the spine, the sensitivity of the culture was $66.80 \%(145 / 217)$ in the treated cases. Although there is no data regarding the incidence of atypical clinical features in patients with tuberculosis of the spine, 47 (32.4\%) patients in our study presented with atypical clinical features, suggesting that one in every three patient with tuberculosis can exhibit an atypical clinical presentation. Currently, MRI is the most popular tool used for the diagnosis of tuberculosis of the spine with good to excellent sensitivity $[7,8]$. However, atypical MRI features have been 
reported in literature to range from $10 \%$ to $25 \%$ in cases of tuberculosis of the spine [19]. Polley and Dunn [19] reported atypical MRI presentation in $16.3 \%$ of the 98 patients from a single surgeon series, with a higher incidence of neurological symptoms in these cases. In our study, an atypical MRI picture was observed in 22 patients (15.17\%). In addition, GeneXpert was used in 46 cases, including 23 tissue samples obtained from an intraoperative biopsy, 18 acquired via a transpedicular biopsy, 3 from the aspiration of a cold abscess, and 1 from a CT-guided and open biopsy. Although the sensitivity of GeneXpert was very high at 95.8\% (23/24) for tissue samples taken during surgery or open biopsy from diseased vertebra and disc material, the sensitivity was reduced to $90.90 \%$ (20/22) for tissue samples obtained via a percutaneous biopsy. Moreover, the overall sensitivity was $93.4 \%$ (43/46), and the sensitivity of GeneXpert to detect rifampicin resistance was $100 \%(7 / 7)$. Furthermore, the rate of rifampicin resistance was $4.8 \%(7 / 145)$ in our study. In all cases exhibiting an atypical MRI presentation, GeneXpert was used to make a rapid and accurate diagnosis. By combining MRI with GeneXpert, we were able to achieve a considerably high sensitivity of $97.9 \%$ for the detection of Mtb in cases of tuberculosis of the spine. Recent literature on GeneXpert supports our findings that the sensitivity for the detection of Mtb and rifampicin resistance using this method can be as high as $95 \%$ and $100 \%$, respectively $[10,11]$. However, there are no references in the literature regarding the accuracy of combining MRI and GeneXpert for the diagnosis of tuberculosis of the spine. Although none of the cases that were neurologically intact at presentation exhibited any neurological deterioration following treatment, 70 patients demonstrated complete improvement, 21 patients partially improved, and 10 patients showed no improvement at the final follow-up. Therefore, no improvement overall was only observed in $6.8 \%(10 / 145)$ of the patients, with $14.4 \%(21 / 145)$ exhibiting a partial improvement, and $79.62 \%(114 / 145)$ with a complete neurological improvement following treatment using this diagnostic protocol.

\section{Conclusions}

An accurate clinical diagnosis of tuberculosis of the spine requires an extremely high degree of clinical suspicion as one in every three patient can exhibit an atypical clinical presentation. In our present study, $32.4 \%$ of patients had an atypical clinical presentation. A culture of acid-fast bacilli in osteoarticular tuberculous lesions remains the gold standard diagnostic test, but it is far from being an ideal screening tool to diagnose tuberculosis of the spine because of its low sensitivity ( $66.80 \%$ in our study). Moreover, MRI is a good screening tool, but $15.17 \%$ of cases in our study exhibited an atypical MRI presentation. In contrast, the sensitivity of GeneXpert for detecting Mtb and rifampicin resistance is excellent. In our study, GeneXpert had a sensitivity of $93.4 \%$ for detecting Mtb and was had a sensitivity of $100 \%$ for predicting rifampicin resistance. Combining MRI with GeneXpert, particularly in cases with an atypical presentation provides a rapid and highly sensitive diagnosis tool to detect both Mtb and rifampicin resistance in patients with tuberculosis of the spine. The use of GeneXpert in conjunction with MRI was $97.9 \%$ sensitive for the diagnosis of tuberculosis of the spine in our study.

\section{Conflict of Interest}

No potential conflict of interest relevant to this article was reported.

\section{Reference}

1. Tuli SM. Tuberculosis of the skeletal system: bones, joints, spine and bursal sheaths. New Delhi: Jaypee Brothers Pvt. Ltd.; 2010.

2. Kumar M, Kumar R, Srivastava AK, et al. Sensitivity of PCR IS6110 in relation to culture and staining in Pott's disease. Indian J Neurosurg 2013;2:46-51.

3. Singh UB, Bhanu NV, Suresh VN, Arora J, Rana T, Seth P. Utility of polymerase chain reaction in diagnosis of tuberculosis from samples of bone marrow aspirate. Am J Trop Med Hyg 2006;75:960-3.

4. Negi SS, Gupta S, Khare S, Lal S. Comparison of various microbiological tests including polymerase chain reaction for the diagnosis of osteoarticular tuberculosis. Indian J Med Microbiol 2005;23:245-8.

5. Pandey V, Chawla K, Acharya K, Rao S, Rao S. The role of polymerase chain reaction in the management of osteoarticular tuberculosis. Int Orthop 2009;33: 801-5.

6. Griffith JF, Kumta SM, Leung PC, Cheng JC, Chow LT, Metreweli C. Imaging of musculoskeletal tuberculosis: a new look at an old disease. Clin Orthop 
Relat Res 2002;(398):32-9..

7. Danchaivijitr N, Temram S, Thepmongkhol K, Chiewvit P. Diagnostic accuracy of MR imaging in tuberculous spondylitis. J Med Assoc Thai 2007;90: 1581-9.

8. Jain AK, Sreenivasan R, Saini NS, Kumar S, Jain S, Dhammi IK. Magnetic resonance evaluation of tubercular lesion in spine. Int Orthop 2012;36:261-9.

9. Yapici F, Atici Y, Balioglu MB, et al. A comparison of two techniques: open and percutaneous biopsies of thoracolumbar vertebral body lesions. J Craniovertebr Junction Spine 2015;6:36-9.

10. Held M, Laubscher M, Zar HJ, Dunn RN. GeneXpert polymerase chain reaction for spinal tuberculosis: an accurate and rapid diagnostic test. Bone Joint J 2014; 96B:1366-9.

11. Yagmur G, Albayrak N, Das T, Yildirim M, Ozgun A, Buyuk Y. Comparison of two different real-time PCR systems in postmortem diagnosis of tuberculosis in paraffin-embedded tissues. Mikrobiyol Bul 2014;48: 577-84.

12. Maynard FM Jr, Bracken MB, Creasey G, et al. Inter- national Standards for Neurological and Functional Classification of Spinal Cord Injury: American Spinal Injury Association. Spinal Cord 1997;35:266-74.

13. Jain AK. Tuberculosis of the spine. Clin Orthop Relat Res 2007;460:2-3.

14. Garg RK, Raut T, Malhotra HS, et al. Evaluation of prognostic factors in medically treated patients of spinal tuberculosis. Rheumatol Int 2013;33:3009-15.

15. Kramer N, Rosenstein ED. Rheumatologic manifestations of tuberculosis. Bull Rheum Dis 1997;46:5-8.

16. Van der Spoel van Dijk A, A MC, Botha PL, Shipley JA, Kapnoudhis MA, Beukes CA. The diagnosis of skeletal tuberculosis by polymerase chain reaction. Cent Afr J Med 2000;46:144-9.

17. Agrawal V, Patgaonkar PR, Nagariya SP. Tuberculosis of spine. J Craniovertebr Junction Spine 2010;1:7485.

18. Garg RK, Somvanshi DS. Spinal tuberculosis: a review. J Spinal Cord Med 2011;34:440-54.

19. Polley P, Dunn R. Noncontiguous spinal tuberculosis: incidence and management. Eur Spine J 2009;18: 1096-101. 